\title{
Importance of Calibration of Photos Obtained by A Smartphone in Facial Prosthesis
}

\author{
Mendes Ariane Ali Bento*1, Seelaus, Rosemary ${ }^{2}$, Salazar-Gamarra Rodrigo ${ }^{3}$ and Dib Luciano \\ Lauria $^{4}$ \\ ${ }^{1}$ Master Student-Paulista University (UNIP), São Paulo, Brazil
}

${ }^{2}$ CCA, MAMS-University of Illinois at Chicago, USA

${ }^{3} D D S, M S c$, PhD-Postgraduate Program Paulista University (UNIP), São Paulo, Brazil

${ }^{4}$ DDS, PhD-Professor Postgraduate Program, Paulista University (UNIP), São Paulo, Brazil

*Corresponding author: Mendes Ariane Ali Bento, Paulista University (UNIP), São Paulo, Brazil, Rua Afonso Braz, 525 - Cj. 81 Vila

Nova Conceição - São Paulo/SP Brasil

\section{ARTICLE INFO}

Received: 蔧 March 23, 2021

Published: April 05, 2021

Citation: Mendes Ariane Ali Bento, Seelaus, Rosemary, Salazar-Gamarra Rodrigo, Dib Luciano Lauria. Importance of Calibration of Photos Obtained by A Smartphone in Facial Prosthesis. Biomed J Sci \& Tech Res 35(1)-2021. BJSTR. MS.ID.005630.

Keywords: Calibration of Photos; Facial Prosthesis; Smartphone; Color of Patient's Skin

\section{ABSTRACT}

Purpose: Aiming at the difficulty of manufacturing a facial prosthesis that has the exact color of the patient's skin, the proposal of this study was to evaluate the color difference between photographs obtained using a smartphone with and without calibration in relation to a reference element, in order to standardize the skin tone used in facial prostheses.

Methods: We used a Samsung Galaxy S8+ smartphone having two polarized light filters that were positioned perpendicularly on the camera and flash, and the reference element, which is an A2 color sample of the VITA-PAN color scale. Three patients were photographed carrying a facial prosthesis and holding the reference element over their faces. Using the software program Adobe PhotoShopCC, the photographs were calibrated according to the known $\mathrm{L}^{*} \mathrm{a}^{*} \mathrm{~b}^{*}$ values of the A2 color sample, and five points were selected on the original photograph and the photograph was calibrated for comparison, totaling 30 points. The tonalities were compared numerically according to the formula $\Delta E=\left[\left(L_{1}-L_{2}\right)^{2}+\left(a_{1}-a_{2}\right)^{2}+\left(b_{1}-b_{2}\right)^{2}\right] / 2$. The intraclass correlation test was applied to determine the concordance between the photos before and after calibration. Furthermore, the Student t-test for paired samples was applied to determine differences in the mean values of the studied parameters before and after calibration. The statistical program SPSS 2.1 was used, adopting $\alpha=0.05$.

Results: In relation to the editing of the photographs, the statistical analysis revealed a significant difference in $\mathrm{L}^{*}$ and $\mathrm{a}^{*}$ values before and after calibration, while in the $\mathrm{b}^{*}$ value, there was no significant change in color.

Conclusion: Based on the results, it is concluded that the proposed standardization technique, using polarized light filters and calibration with a reference element, of photographs obtained using a smartphone camera is appropriate for distinguishing colors in the production of facial prostheses.

Clinical Implication: This new methodology can be used to increase the reliability of the smartphone as a tool for the manufacture of the facial prosthesis.

\section{Introduction}

Facial mutilations cause aesthetic, functional, and psychological alterations that can affect quality of life and lead to social isolation $[1,2]$. For the rehabilitation of facial defects, surgical procedures or facial prosthetics can be used to restore the facial anatomy, depending on the extent of the defect, the patient's age, complications related to radiotherapy, and the patient's medical 
condition [3-5]. Facial prostheses are made from silicone, due to its excellent properties of flexibility, texture, and color, which are similar to those of natural skin [6]. However, one of the main difficulties in the manufacture of such prostheses is obtaining a color tone that closely matches color tone of the patient's own skin. Color is a response to the physical interaction of light energy with an object and the subjective experience of an observer, being characterized as a psychophysical event [7]. Color can be defined as the appearance of any object that can be reproduced in terms of hue, luminosity, and saturation. The Commission Internacionale de l'Eclairage created the $\mathrm{L}^{*} \mathrm{a}^{*} \mathrm{~b}^{*}$ color system in 1974 . The system identifies the colors numerically, whereby "L" is the brightness coordinate (ranging from 0 to 100 on a scale from darker to lighter tone), "a" is the red-green coordinate (the higher "a" is, the redder the hue, and the lower "a" is, the greener the hue) and "b" is the yellow-blue coordinate (the higher " $b$ " is, the yellowish the hue, and the lower " $b$ " is, the bluer the hue) [8].

The color of an object can be captured both instrumentally and visually [9]. By the instrumental method, a high-precision device called a spectrophotometer can be used to obtain the correct data on the tonality to be captured. This method has the advantage of being more decisive, and possible environmental interference in the color capture can be controlled. However, this equipment is costly. The visual method is the one currently used for the manufacture of facial prostheses and involves comparison of the color of an object with a color scale or reference. It is a simple method, but it is susceptible to factors that can affect the correct color recognition, such as the brightness of an object, the professional's experience and age, vision fatigue, and color blindness [10]. This discrepancy in capturing the actual color of the patient's skin can lead to errors, with the prosthesis having to be remade, incurring higher material cost and a longer time to complete the work. These discrepancies may be even more important when 3D digital workflows, which include 3D models of faces obtained by optical resources, will evolve into color 3D printing for final facial prosthesis delivery, like the PlusID (+ID) methodology which uses smartphones and "UV maps" from monoscopic photogrammetry to obtain the color information of the face of the patient $[11,12]$. In view of the high cost of these instruments for accurate color capture, they are not widely used in rehabilitation centers, especially in those with fewer investments. Therefore, it is important to develop research to produce devices for capturing color that are lower in cost and easier to use [13]. Recent studies have used a smartphone application as a colorimeter, which has proven to be a viable way to capture color for facial prostheses. However, they highlight the need for new research for image calibration, in order to increase reliability and control variables such as lighting and other environmental interferences [14]. Therefore, the present study proposes to use a smartphone to obtain photographs and develop a digital calibration method to increase the reliability of obtaining the real color, in order to improve color reproducibility in facial prostheses.

\section{Methods}

This study was carried out at the Dentistry Clinic of Paulista University, UNIP, with the approval of the Research Ethics Committee (CAAE) 10833119.4.0000.5512, opinion no. 3,359,029, after obtaining a signed informed consent form from the patients.

\section{Patient Selection}

Among the 19 patients undergoing treatment at the Maxillofacial Rehabilitation Clinic of the UNIP in 2018, patients with unilateral eyelid defects and had used an oculo-facial prosthesis for one month or less were selected. Those with a facial defect extending beyond the eyelid margin were excluded. Thus, three patients were selected: two female and one male, aged between 30 and 65 years, and self-reported as White.

\section{Lighting and Ambience}

Ambient light, inside the clinic, was guaranteed only by nonincident natural lighting directly on the patient's face, with the face illuminated naturally, without any exacerbated brightness. The patient was positioned in front of a white surface, as a bachground, without any markings, with the face turned directly toward the camera.

\section{Standardization}

The glow of the photographs was standardized using two polarized light filters - Lee Filters, which were positioned with their axes perpendicular to each other having one light filter over the camera of the cell phone and the other over the flash, fixed with adhesive tape. The polarized light filter has a linear filtering pattern; when the linear patterns from the source of light emission (the camera flash) and the light receptor (the camera) are crossed, the glow of the photograph is filtered (Figure 1). For the color standardization, the patient wasjp office instructed to hold an A2 color sample of the VITA-PAN Color Scale over the lip or nose. This color sample was used as a calibration element, since the real colors shown on the chart have been previously defined by the EasyShade V spectrophotometer, VITA Zahnfabrik $\left(\mathrm{L}^{*}=83, \mathrm{a}^{*}=1\right.$, and $\mathrm{b}^{*}=22$, according to coordinates $\mathrm{L}^{*} \mathrm{a}^{*} \mathrm{~b}^{*}$ ), independent of environmental factors. This element was later used in Adobe Photoshop to calibrate the color of the entire photograph. 


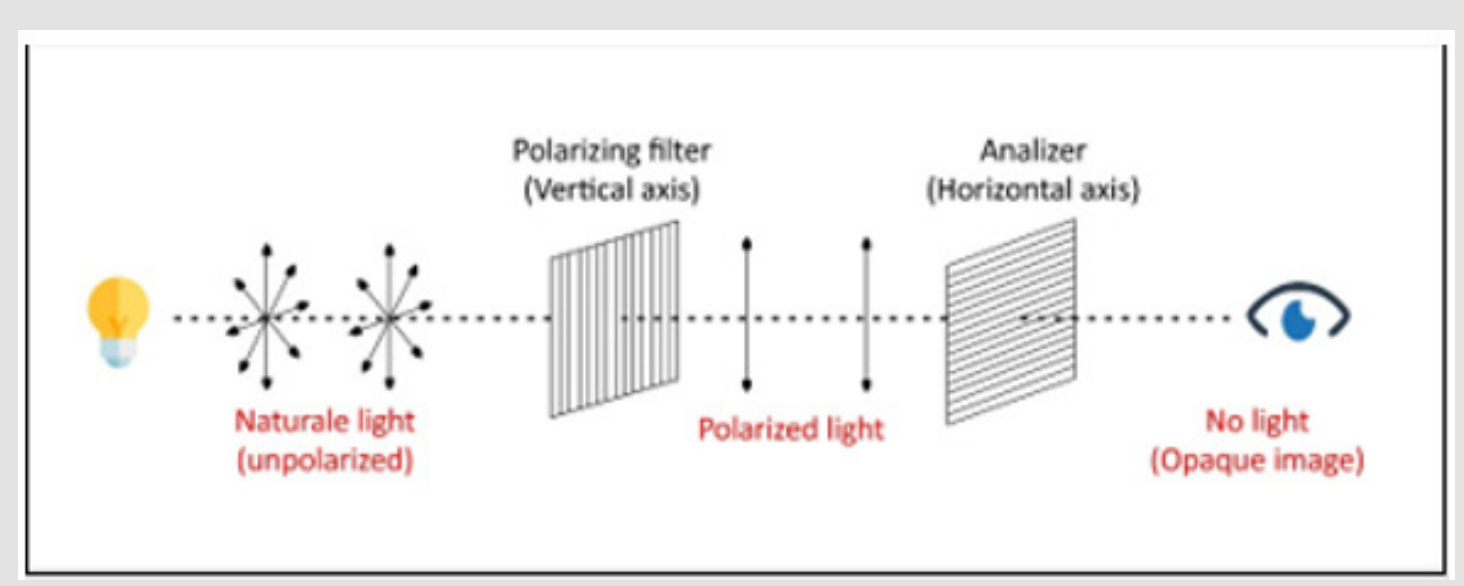

Figure 1: Huygens' principle in the application of crossed polarized light filters. Adapted from CESAD - UFS.

\section{Photographic Shots}

Photographic shots of each patient were taken using a Samsung Galaxy S8+ smartphone, Seoul, South Korea in automatic mode and flash on, to minimize environmental interferences. The patients were photographed according to the American Board of Orthodontics (ABO) standard: in portrait, including the tops of the shoulders and the entire face, with all parts of the face in focus. The patient's face was turned directly toward the camera, with the eyes open, teeth in occlusion, lips lightly closed, and the ears visible. The patient's head was oriented on the sagittal, axial, and bipupillary planes, parallel to the edges of the photograph, so that the tip of the patient's nose was approximately in the center of the photograph. The distance between the professional and the patient was one and a half meters (Figure 2).

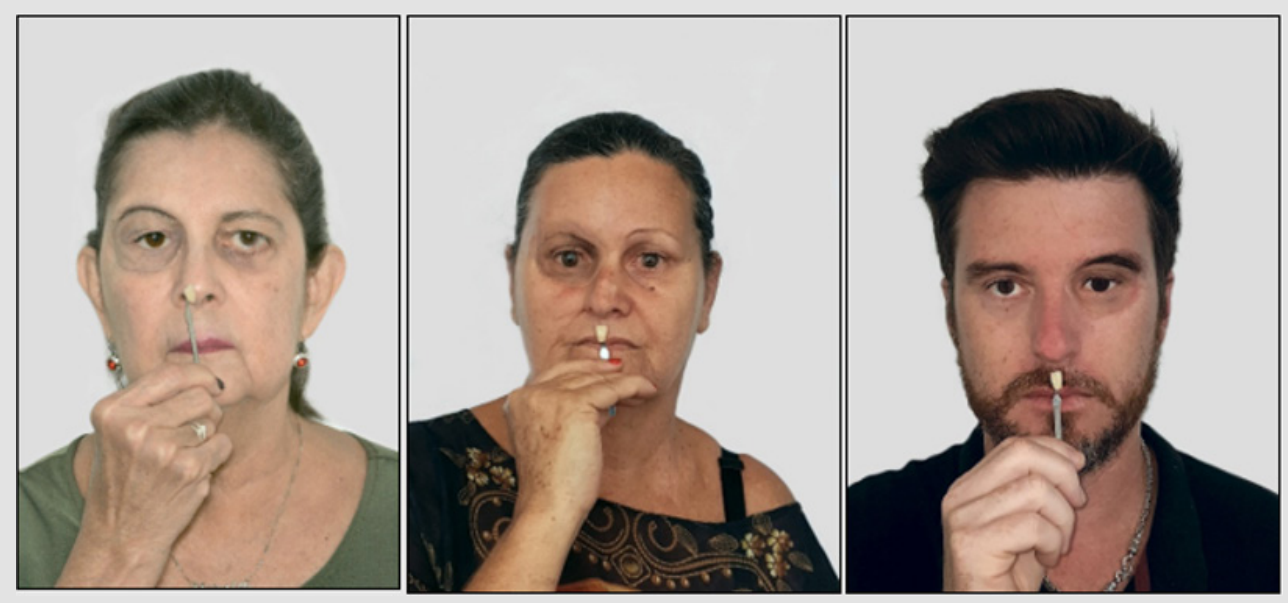

Figure 2: Patients wearing a facial prosthesis positioned according to the $\mathrm{ABO}$, holding the $\mathrm{A} 2$ color sample over the nose or lip.

\section{Digital Calibration Method}

Using the software program Adobe Photoshop CC, version 20.0.6, we calibrated the photos by adjusting the $\mathrm{L}^{*} \mathrm{a}^{*} \mathrm{~b}^{*}$ values of the VITA sample of the original photo, matching them with the $\mathrm{L}^{*} \mathrm{a}^{*} \mathrm{~b}^{*}$ of the VITA sample determined previously by Easy Shade. With this maneuver, we obtained a new photograph called a "calibrated photograph," with a new definition of colors, which became the real colors, regardless of any changes in brightness, environmental conditions, and luminosity, such as interference in the clinical environment.

\section{Calculating the color variation after calibrating the photographs}

To compare the color difference between the original and the calibrated photographs, we determined points of analysis between the patient's skin and portions of the prostheses. These points of comparison between the healthy skin and the facial prosthesis were determined based on the vertical and horizontal axes, coinciding with the end of the prosthesis and its deepest and most superficial points (total of five symmetrical points) (Figure 3). The distance of these points from the pupil, was measured and then transferred to 
the healthy portion of the patient's photograph, in order to make the points symmetrical. If there was a spot or mole on the patient's skin, the collection point was slightly diverted to make the skin tone less discrepant. Having established the points, the $\mathrm{L}^{*} \mathrm{a}^{*} \mathrm{~b}^{*}$ values of each point were captured using the eyedropper tool of Adobe Photoshop CC. These values were recorded in a table to calculate the difference in color. The symmetrical points were determined on the calibrated and original photographs, obtaining 20 points in total, per patient. Four groups were established: Prosthesis with Calibration (PwC group), Eye with Calibration (EwC), Prosthesis without Calibration (Pw/oC) and Eye without Calibration (Ew/oC). The first two referred to "calibrated photographs" and the last two referred to "original photographs." Each group had five points, i.e., $5 \mathrm{~L}^{*} \mathrm{a} \mathrm{b}^{*}$ coordinates.

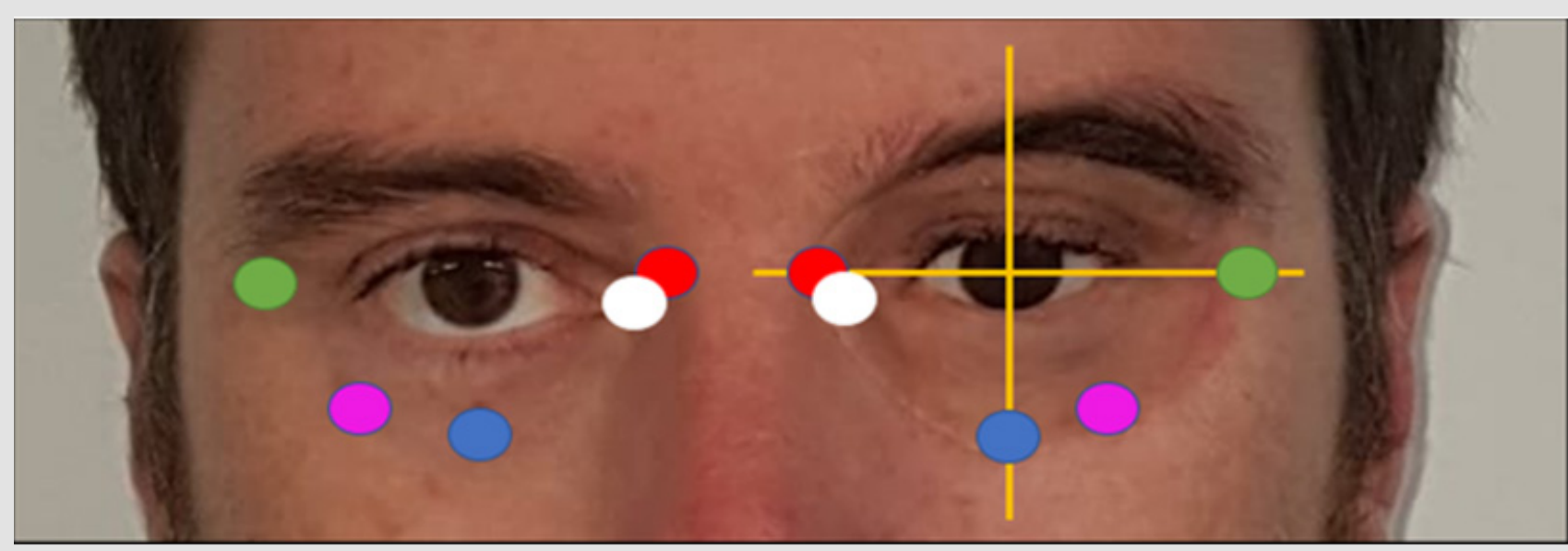

Figure 3: Color collection points on the facial prosthesis and the patient's skin, taking the pupil as reference.

\section{Calculating the Difference in Tonality}

For the calculation of numerical difference in tonality between the colors obtained, the formula of difference in tonality was applied [15]:

$$
\Delta E=\left[\left(L_{1}-L_{2}\right)^{2}+\left(a_{1}-a_{2}\right)^{2}+\left(b_{1}-b_{2}\right)^{2}\right] / 2 .
$$

The $\mathrm{L}^{*} \mathrm{a}$ b values of each point and their respective symmetrical correspondents were inserted into the formula, so that at the end of the calculation the $\Delta \mathrm{E}$ value was obtained, which is equivalent to the numerical difference between the two tonalities. The first calculation was between the $\mathrm{Pw} / \mathrm{oC}$ and $\mathrm{PwC}$ groups, aiming to evaluate the color variation before and after calibration on the patient's prosthesis. The next groups to be submitted to the tonality difference formula were $\mathrm{Ew} / \mathrm{oC}$ and $\mathrm{EwC}$, to evaluate the variation in the patient's skin tone before and after calibration. Groups of the same photograph were also compared; the $\mathrm{Pw} / \mathrm{oC}$ and $\mathrm{Ew} / \mathrm{oC}$ groups, and the $\mathrm{PwC}$ and $\mathrm{EwC}$ groups.

\section{Statistical Analysis}

The intra class correlation test (ICC) was applied to verify concordance between photos before calibrating and after calibration. Posteriorly, the t-Student test for paired samples was applied, with the aim of verifying difference in the averages of the parameters studied before and after calibration. Was the statistical program SPSS 2.1 was used, adopting $\alpha=0.05$.

\section{Results}

For the analysis of concordance between the points collected without calibration and after calibration, the intraclass agreement test was applied to the points without calibration and after calibration, as shown in Table 1. The collection of points from the five different regions, both on the prosthesis and on the eyelid (inner edge, outer edge, lower edge, most superficial, and deepest point) resulted in 30 measurements. The result of the intra class correlation showed a correlation between the parameters analyzed in the groups $(\mathrm{p}<0.05)$. The $\mathrm{L}^{*}, \mathrm{a}^{*}$, and $\mathrm{b}^{*}$ parameters for the prosthesis in the photographs before calibration and after calibration were considered adequate (ICC $<0.75$ ), demonstrating that they are comparable. An adequate ICC in the eye region was also found for the $\mathrm{L}^{*}$ and $\mathrm{a}^{*}$ parameters, with ICC $=0.910$ and 0.960 , respectively $(p<0.05)$. For the $b^{*}$ parameter, the ICC was 0.67 , which is considered moderate, but significant. After the analysis of concordance, the parametric test was applied to compare paired Student $\mathrm{t}$ mean values. The results are shown in Table 2 \& Graph 1. The results showed a significant difference between the mean color values for the $\mathrm{L}^{*}$ and $\mathrm{a}^{*}$ parameters (in both the prosthesis and the eye) when comparing the photographs before calibration and after calibration. In the $\mathrm{L}^{*}$ parameter after calibration, a significant increase in the mean values of the calibrated photos was observed when compared before calibration, both in the prosthesis and in the eye. For the $a^{*}$ parameter after calibration, there was a significant reduction in mean values when compared to the photos 
before calibration. However, no significant differences were observed in the $b^{*}$ parameter when comparing before and after calibration.

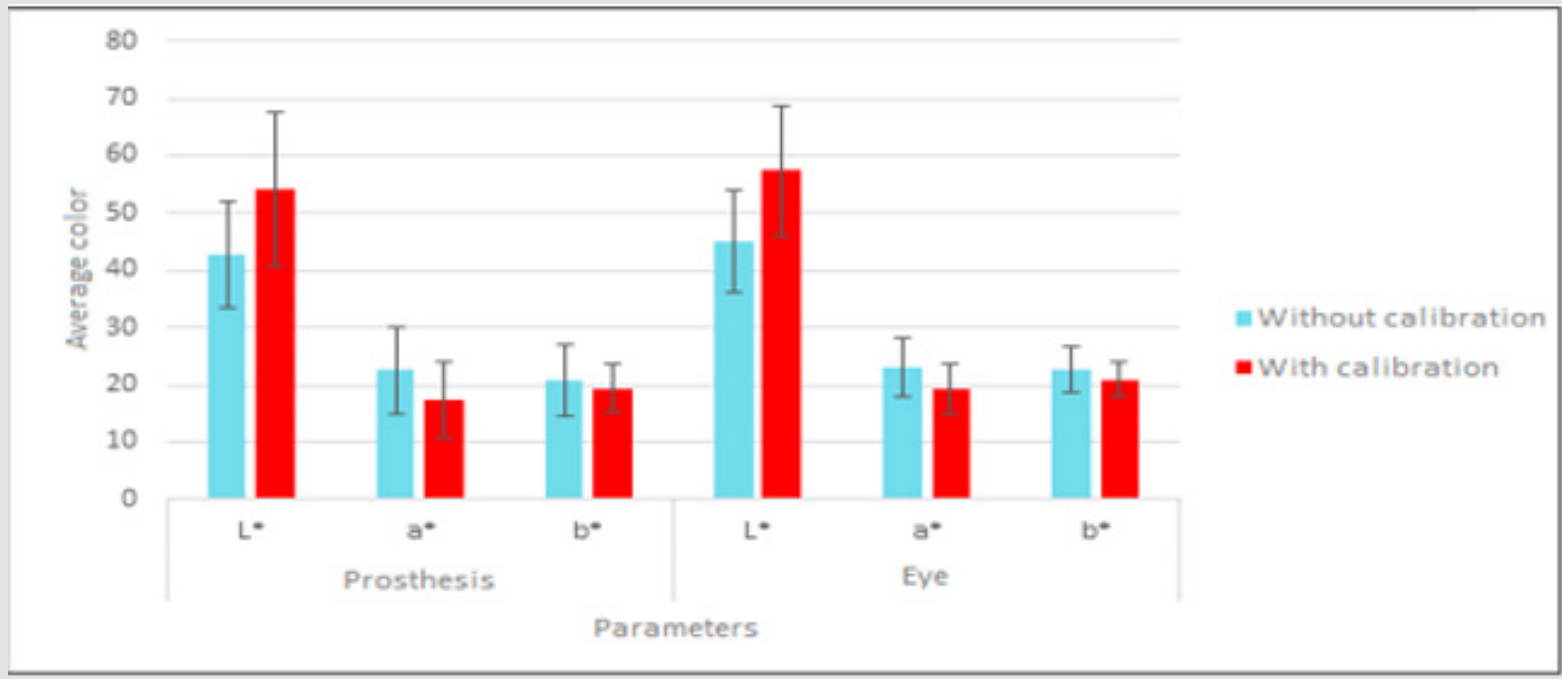

Graph 1: Average color.

Table 1: Color parameters in the eyes and prosthesis.

\begin{tabular}{|c|c|c|c|c|}
\hline Type & $\begin{array}{c}\text { Color } \\
\text { parameter }\end{array}$ & Groups & ICC & p-valor \\
\hline \multirow{6}{*}{ prosthesis } & \multirow{2}{*}{$L^{*}$} & Without calibration & \multirow{2}{*}{0,939} & \multirow{2}{*}{0,000} \\
\hline & & With calibration & & \\
\hline & \multirow{2}{*}{$a^{*}$} & Without calibration & \multirow{2}{*}{0,990} & \multirow{2}{*}{0,000} \\
\hline & & With calibration & & \\
\hline & \multirow{2}{*}{$\mathrm{b}^{*}$} & Without calibration & \multirow{2}{*}{0,903} & \multirow{2}{*}{0,000} \\
\hline & & With calibration & & \\
\hline \multirow{6}{*}{ Eye } & \multirow{2}{*}{$\mathrm{L}^{*}$} & Without calibration & \multirow{2}{*}{0,910} & \multirow{2}{*}{0,000} \\
\hline & & With calibration & & \\
\hline & \multirow{2}{*}{$a^{*}$} & Without calibration & \multirow{2}{*}{0,960} & \multirow{2}{*}{0,000} \\
\hline & & With calibration & & \\
\hline & \multirow{2}{*}{$\mathrm{b}^{*}$} & Without calibration & \multirow{2}{*}{0,674} & \multirow{2}{*}{0,022} \\
\hline & & With calibration & & \\
\hline
\end{tabular}

Table 2: Color parameter with Student.

\begin{tabular}{|c|c|c|c|}
\hline \multirow{2}{*}{ Type } & $\begin{array}{c}\text { Color } \\
\text { parameter }\end{array}$ & $\begin{array}{c}\text { Without } \\
\text { Calibration }\end{array}$ & $\begin{array}{c}\text { With } \\
\text { Calibration }\end{array}$ \\
\hline \multirow{3}{*}{ Prosthesis } & $\mathrm{L}^{*}$ & $42,87(9,3)^{*}$ & $54,07(13,3)$ \\
\cline { 2 - 4 } & $\mathrm{a}^{*}$ & $22,47(7,4)^{*}$ & $17,47(6,6)$ \\
\cline { 2 - 4 } & $\mathrm{b}^{*}$ & $20,67(6,3)$ & $19,33(4,1)$ \\
\hline \multirow{3}{*}{ Eye } & $\mathrm{L}^{*}$ & $45,07(8,8)^{*}$ & $57,40(11,3)$ \\
\cline { 2 - 4 } & $\mathrm{a}^{*}$ & $23,07(5,2)^{*}$ & $19,20(4,3)$ \\
\cline { 2 - 4 } & $\mathrm{b}^{*}$ & $22,60(3,9)$ & $20,87(3,0)$ \\
\hline
\end{tabular}

Note: *Indicates a statistically significant difference between the groups compared.

\section{Discussion}

The smartphone has recently been used in studies in the area of facial prostheses to reproduce the facial anatomy through photogrammetry and texture maps, seeking to refine the definition of the patient's skin $[11,12]$. The smartphone has also been used as a colorimeter in color studies in facial prosthesis, but there is a need to increase the reliability of methods for obtaining color through the cameras of these devices [14]. Staining is one of the most complex stages in the manufacture of facial prostheses, as small changes in tonality can be perceived by the human eye, which has the same ability to identify colors as colorimeters and spectrophotometers, so that even minimal differences in tonality between the skin and the prosthetics are noticeable to the patients and to those around them [16]. Thus, considering that the smartphone is a versatile tool, with potential use in the manufacture of facial prostheses, this study aimed to find alternative ways to enable its use in obtaining and standardizing colors. Previous research has shown that various environmental factors, such as lighting, skin brightness, shadows, and camera configuration, generate interference and modify the accuracy of the photographs obtained [14]. To control these factors, the present study uses a 3-step method of calibration of the photographs: the use of filters on the lens and flash of the camera; the use of a reference element for editing images in software; and the definition of points for color comparison between the prosthesis and the skin.

In the first stage of calibration, a cross-polarized light filter was placed over the lens and flash of the smartphone camera, to filter any kind of brightness in the photograph, regardless of the amount of light exposure on the patient's face. To enable the photographs to be edited in Adobe Photoshop CC, photographic shots were taken of the patients holding a reference element, the $\mathrm{L}^{*} \mathrm{a}^{*} \mathrm{~b}^{*}$ color values of which had been previously determined using a spectrophotometer. Thus, in order to create a photograph that was calibrated by the software, the $\mathrm{L}^{*} \mathrm{a}^{*} \mathrm{~b}^{*}$ values of the reference element of the original photograph were modified, matching them with the known values, generating the modification of colors across the entire photograph. 
To be able to compare whether the original and calibrated photos were different, five points were selected on the prosthesis and five points on the patient's healthy skin. This selection sought to create a wide sample of comparisons of color nuances and was based on the different shades of patients' skins, as well as on the natural difficulty of reproducing these variations in the different areas of skin. To evaluate whether the photographs could be compared with each other, an intraclass agreement test was applied. This test demonstrated that the parameters were adequate, making this comparison possible. To observe the numerical differences between the photographs before and after calibration, the Student t-parametric test was applied, which showed significant differences between the tonalities of both photographs. The interpretation of these results showed that the original photograph presented great color distortion with statistically significant differences, when compared to the colors obtained in the calibrated photograph. The latter can be considered as the real color, since the $\mathrm{L}^{*} \mathrm{a}$ *b values had been predetermined.

The results of this present study are in agreement with the literature, which suggests a need to calibrate smartphone cameras for possible use in photogrammetry. There currently exists an additive manufacturing technology capable of printing prostheses on a type of colored resin (Polyjet Stratasys, Rehovot) after applying a specific workflow. This technology can be used as an immediate or temporary alternative for cases of extensive facial resections; however, reproducing real color is one of the greatest challenges for the effective production of this fully printed 3D prosthesis. This fact reinforces the importance of this study in the context of calibration and standardization of colors [15]. With the methodology proposed in this study, the calibration of photographs with a simple reference element can allow the professional to use any model of smartphone without having to use complex camera settings, but only automatic mode given that standardization with filters and calibration can ensure the authenticity of the colors. In contrast to expensive spectrophotometry equipment, the smartphone is a simple and affordable resource that is easy to handle, and is already used for different functions in the production of facial prostheses. Based on the method of standardization and calibration of photographs proposed in this study, its use can be expanded, taking another step toward the production of a fully digital prosthesis. New studies are needed to put this methodology to the test, evaluating variations in skin tones and the accuracy of obtaining silicone samples compatible with the colors obtained from the patient's skin through this technique. In natural evolution of knowledge and 3D printing technology, with silicone printers of the future after defining the patient's actual skin tone using a smartphone, it will be possible to print a prosthesis without the need for retouching by a human hand. However, even before such silicone printing capabilities are developed, it will be possible to use this technique to define actual skin color and compare with silicone samples developed by prosthesis specialists, with a higher degree of precision, reducing working time, and the need for costly equipment.
Within the limitations of the small sample size of this study, the results show that standardization of photographs using polarized light filters, and calibration of colors using a reference element, enable the use of the smartphone as a tool for obtaining and defining colors for the production of facial prostheses. Future studies with a higher number of samples are needed to prove our results. Comparison between the pre- and post-calibration photographs showed statistically significant difference, demonstrating that without standardization or calibration, the smartphone is not a reliable tool for color definition; its use being restricted to documenting of cases, but not extending to the production of prostheses. The proposed technique, which includes the usage of smartphones, provides a reliable and accessible workflow to assist the specialist to obtain calibrated digital photography to register different regions of the facial skin and facial prosthesis. Reduced discrepancies between the real color of a face and the output file of a digital photography is an important tool for quality control, as well as in manufacturing process. In the near future, when digital workflows of Color 3D Printing may be predictable to transform smartphone captures up to the final colored 3D prosthesis, color calibration will be one of the most important steps for an accurate fabrication.

\section{Conclusion}

The results showed that with the proposed standardization technique using polarized light filters and calibration of photographs against a reference element, a smartphone camera can be accurately used to distinguish colors for the production of facial prostheses. Further studies are needed to corroborate our results and expand the potential of the smartphone, with the aim of improving the esthetics of prostheses and optimizing clinical time for their manufacture.

\section{References}

1. Atay A, Peker K, Günay Y, Ebrinç S, Karayazgan B, et al. (2013) Assessment of health-related quality of life in Turkish patients with facial prostheses. Health and Quality of Life Outcomes 11(1): 1-9.

2. De Oliveira FM, Salazar-Gamarra R, Öhman D, Nannmark U, Pecorari V, et al. (2018) Quality of life assessment of patients utilizing orbital implantsupported prostheses. Clinical Implant Dentistry and Related Research 20(4): 438-443.

3. B Jamayet N, J Abdullah Y, A Rajion Z, Husein A, K Alam M (2017) New Approach to 3D Printing of Facial Prostheses Using Combination of Open Source Software and Conventional Techniques: A Case Report. The Bulletin of Tokyo Dental College 58(2): 117-124.

4. Thiele OC, Brom J, Dunsche A, Ehrenfeld M, Federspil P, et al. (2015) The current state of facial prosthetics - A multicenter analysis. Journal of Cranio-Maxillofacial Surgery 43(7): 1038-1041.

5. Thiele OC, Mertens C, Bacon C, Flechtenmacher C, Zaoui K, et al. (2014) Facial basal cell carcinoma with successive metastases to the neck, thyroid gland and lung. Journal of Cranio-Maxillofacial Surgery 42(5): 489-491.

6. Kim SM, Cho YJ, Eo MY, Kim JS, Lee SK (2017) Silicone Facial Prosthesis. Journal of Craniofacial Surgery 00(00): 1.

7. Bridgeman I (1987) The nature of light and its interaction with matter. Colourphysics for Industry, p. 1-34. 
8. Committee CM (n.d.) Uniform Colour Space and Colour-difference Formula. XIII-The Development of the CIE 1976 (L " a “ b ").

9. Alshiddi IF, Richards LC (2015) A comparison of conventional visual and spectrophotometric shade taking by trained and untrained dental students. Australian Dental Journal 60(2): 176-181.

10. Lasserre JF, Pop-Ciutrila IS, Colosi HA (2011) A comparison between a new visual method of colour matching by intraoral camera and conventional visual and spectrometric methods. Journal of Dentistry 39(3): e29-e36.

11. Salazar-Gamarra R, Moraes CADC, Seelaus R, Dib LL, Ulloa JJ (2019) Introdução à metodologia "mais identidade": próteses faciais $3 \mathrm{~d}$ com a utilização de tecnologias acessíveis para pacientes sobreviventes de câncer no rosto. In Comunicação Científica e Técnica em Odontologia, pp. 251-272.

12. Salazar-Gamarra R, Seelaus R, Da Silva JVL, Da Silva AM, Dib LL (2016) Monoscopic photogrammetry to obtain 3D models by a mobile device:

\section{ISSN: 2574-1241}

DOI: 10.26717/BJSTR.2021.35.005630

Mendes Ariane Ali Bento. Biomed J Sci \& Tech Res

This work is licensed under Creative Commons Attribution 4.0 License

Submission Link: https://biomedres.us/submit-manuscript.php
A method for making facial prostheses. Journal of Otolaryngology-Head and Neck Surgery 45(1): 1-13.

13. Schmidt W (2004) A mini-rapid-scan-spectrophotometer. Journal of Biochemical and Biophysical Methods 58(2): 125-137.

14. Mulcare DC, Coward TJ (2019) Suitability of a Mobile Phone Colorimeter Application for Use as an Objective Aid when Matching Skin Color during the Fabrication of a Maxillofacial Prosthesis. Journal of Prosthodontics 28(8): 934-943.

15. Coward TJ, Seelaus R, Li SY (2008) Computerized color formulation for African-Canadian people requiring facial prostheses: A pilot study. Journal of Prosthodontics 17(4): 327-335.

16. Piérard GE (1998) EEMCO guidance for the assessment of skin colour. Journal of the European Academy of Dermatology and Venereology 10(1): 1-11.

$\begin{array}{ll}\text { BIOMEDICAL } & \text { Assets of Publishing with us } \\ \text { RESEARCHES } & \text { - Global archiving of articles } \\ \text { - Immediate, unrestricted online access }\end{array}$

